\title{
ÉDITORIAL
}

\section{Les personnes compétentes au cour du retour d'expérience !!}

Comme dans toute revue scientifique, notre objectif premier est de permettre aux chercheurs de faire état des progrès qu'ils réalisent et aux professionnels de la radioprotection de se tenir au courant de l'évolution des connaissances et des techniques. Cela nous est toujours apparu comme une condition indispensable à l'amélioration de la protection de l'homme et de l'environnement. Condition indispensable mais non suffisante, c'est pourquoi nous nous sommes aussi fixé comme objectif de fournir à tous, dans le cadre de notre revue, un outil pour faciliter la diffusion du retour d'expérience et des bonnes pratiques.

De nombreux accidents récents dans le monde nous rappellent qu'il convient de garder une attitude de très grande vigilance dans le maniement des «sources». Il y a un an, lors d'un séminaire du réseau ALARA européen, sur «Les bonnes pratiques de radioprotection dans l'industrie et la recherche», l'ensemble des participants a très fortement recommandé la mise en place de «mécanismes nationaux et européens de retour d'expérience permettant de diffuser largement des études de cas et les leçons que l'on peut tirer de l'analyse des incidents et accidents radiologiques".

Dans cet esprit, notre revue ne peut que se féliciter de l'initiative prise par le groupe des personnes compétentes de la SFRP de susciter la mise en place d'un réseau pour sélectionner et analyser des incidents français en vue d'enrichir la formation et la prévention. Il s'agit de mettre à disposition des enseignants et des praticiens des fiches d'analyse anonymes sur les incidents sélectionnés. Notre revue se propose donc d'ouvrir ses pages pour publier ce type d'études de cas.

Afin d'initier le processus, nous publions dès ce numéro la traduction d'une étude de cas britannique. Il nous paraît en effet souhaitable de bénéficier aussi du retour d'expérience des pays voisins et nous ne pouvons que souhaiter la mise en place au niveau européen d'un système d'échange et de traduction des fiches les plus intéressantes d'un point de vue pédagogique.

C. Lefaure

Vice-Président du comité de rédaction 